\title{
Efficacy and safety of intravenous recombinant tissue plasminogen activator in mild ischaemic stroke: a meta-analysis
}

Shoujiang You, ${ }^{1}$ Anubhav Saxena, ${ }^{2}$ Xia Wang, ${ }^{2}$ WeeYong Tan, ${ }^{3}$ Qiao Han, ${ }^{4}$
Yongjun Cao,, 5 Chun-Feng Liu ${ }^{1,5}$

To cite: You S, Saxena A, Wang $X$, et al. Efficacy and safety of intravenous recombinant tissue plasminogen activator in mild ischaemic stroke: a meta-analysis. Stroke and Vascular Neurology 2018;3: e000106. doi:10.1136/svn2017-000106

- Additional material is published online only. To view please visit the journal online (http://dx.doi.org/10.1136/svn2017-000106).

Part of this article was presented at the Asia Pacific Stroke Conference 2017 (Cerebrovasc Dis 2017;44(suppl 1):1-52).

Received 25 July 2017 Revised 14 November 2017 Accepted 15 November 2017 Published Online First 5 January 2018

Check for updates

For numbered affiliations see end of article.

Correspondence to Dr Yongjun Cao; yongjuncao@126.com

\section{ABSTRACT}

The benefits and safety of intravenous recombinant tissue plasminogen activator (IV-tPA) for patients with mild ischaemic stroke (MIS) are still unclear. The objective of this meta-analysis was to evaluate the efficacy and safety of IV-tPA as treatment for patients with MIS. We performed a systematic literature search across MEDLINE, Embase, Central, Global Health and Cumulative Index to Nursing and Allied Health Literature (CINAHL), from inception to 10 November 2016 , to identify all related studies. Where possible, data were pooled for meta-analysis with odds ratio (OR) and corresponding 95\% confidence interval (Cl) using the fixed-effects model. MIS was defined as having National Institutes of Health Stroke Scale score of $\leq 6$. We included seven studies with a total of 1591 patients based on the prespecified inclusion and exclusion criteria. The meta-analysis indicated a high odds of excellent functional outcome based on the modified Rankin Scale or 0xfordshire Handicap Score 0-1 (OR=1.43; 95\% Cl 1.14 to 1.79; $P=0.002, I^{2}=35 \%$ ) in patients treated with IV-tPA compared with those not treated with IV-tPA $(74.8 \%$ vs $67.6 \%$ ). There was a high risk of symptomatic intracranial haemorrhage (sICH) with IV-tPA treatment $(\mathrm{R}=10.13$; $95 \% \mathrm{Cl} 1.93$ to $\left.53.02 ; \mathrm{P}=0.006, \mathrm{I}^{2}=0 \%\right)(1.9 \%$ vs $0.0 \%)$ but not mortality $(\mathrm{OR}=0.78 ; 95 \% \mathrm{Cl} 0.43$ to $1.43 ; \mathrm{P}=0.43$, $\left.\mathrm{I}^{2}=0 \%\right)(2.4 \%$ vs $2.9 \%)$. Treatment with IV-tPA was associated with better functional outcome but not mortality among patients with MIS, although there was an increased risk of sICH. Randomised trials are warranted to confirm these findings.

\section{INTRODUCTION}

Intravenous administration of recombinant tissue plasminogen activator (IV-tPA) remains the only Food and Drug Administration-approved thrombolysis therapy for patients with acute ischaemic stroke within 4.5 hours after symptom onset. Mild ischaemic stroke (MIS) is common ${ }^{1-3}$ and is associated with disability. ${ }^{4-6}$ However, whether patients with MIS can benefit from IV-tPA treatment remains unclear. The latest American Heart Association/American Stroke Association guidelines recommend that IV-tPA treatment may be considered for patients with MIS (class IIb; level of evidence $\mathrm{C})^{7}$; however, no recommendations were found in European, Japan and Canadian guidelines. ${ }^{8-10}$

Existing literature exploring the use of thrombolysis in MIS is controversial. ${ }^{11-22}$ Some studies have shown IV-tPA treatment is associated with excellent outcome,${ }^{11-17}$ while other studies found no benefit from IV-tPA therapy. ${ }^{18-22}$ These inconsistent results may be due to differences in sample size, study design, study population or confounding among studies. Three meta-analyses published in 2014 had investigated IV-tPA treatment in patients with MIS. ${ }^{15} 1722$ However, in these analyses, a limited number of studies were included, the risk of symptomatic intracranial haemorrhage (sICH) was not compared, and the results were conflicting. Hence, we systematically evaluated currently available studies to evaluate the efficacy and safety of IV-tPA treatment in patients with MIS.

\section{METHODS}

\section{Databases and sources}

We identified all published studies assessing the efficacy and safety of thrombolysis for mild stroke using MEDLINE, Embase, Central, Global Health and Cumulative Index to Nursing and Allied Health Literature (CINAHL). The search terms were 'mild OR minor OR mini OR warning' AND 'stroke OR cerebrovascular disease' AND 'thrombolysis OR thrombolytic OR alteplase OR rtPA OR urokinase'; a comprehensive search strategy can be found in the online supplementary file. The search strategy was developed without any language restriction.

\section{Study selection}

Two reviewers (SY and AS) performed the study selection independently, with 


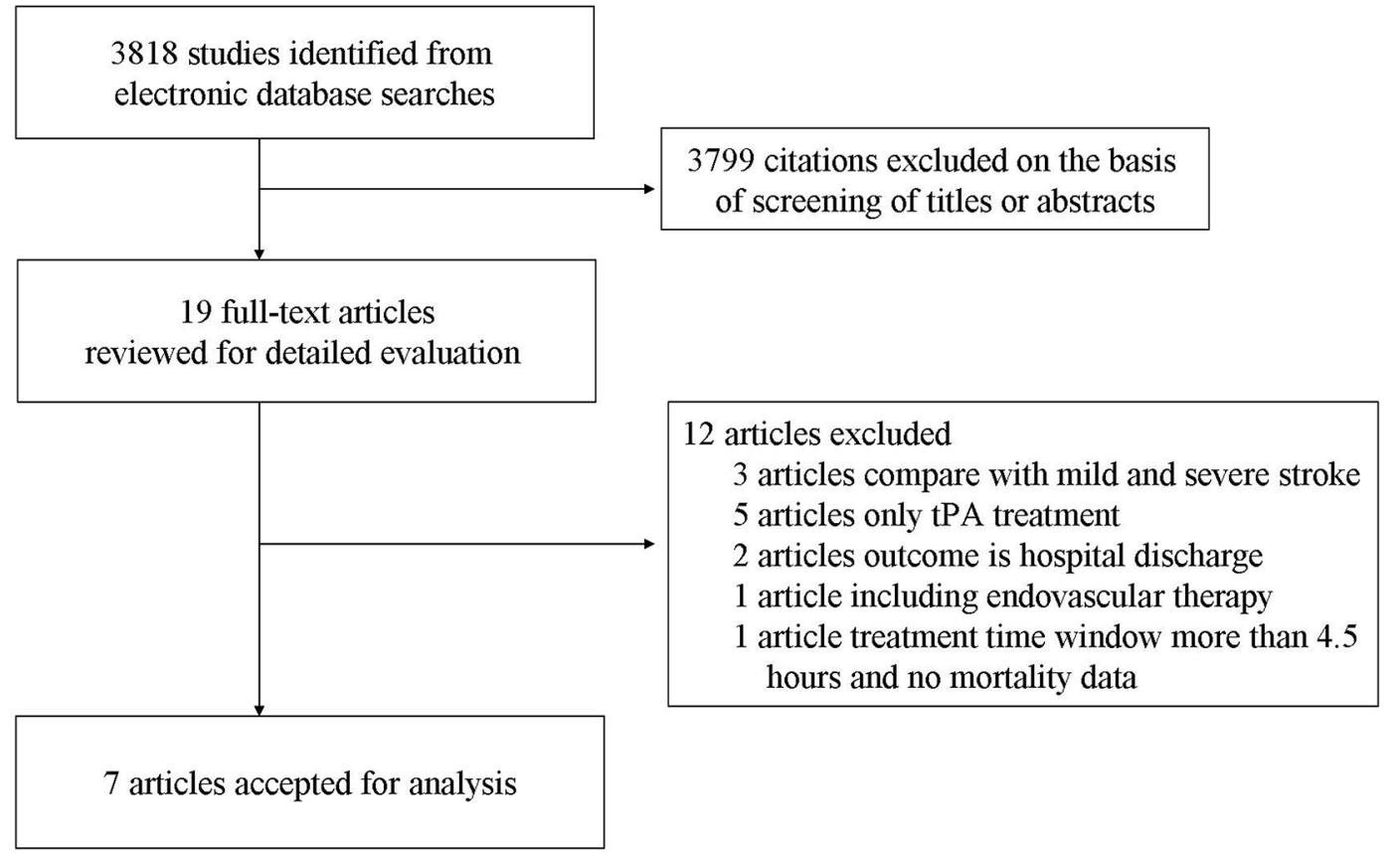

Figure 1 Flow chart of literature screening and selection process. tPA, tissue plasminogen activator.

disagreements resolved through discussions and by consulting a third reviewer (XW), if necessary. Studies were considered potentially eligible for this systematic review if they met the following inclusion criteria: (1) diagnostic criteria for MIS (National Institutes of Health Stroke Scale (NIHSS) score of $\leq 6)$; (2) the study recorded baseline characteristics information, including study type, first author(s), study locations, study period, numbers of total and subgroup subjects, mean age, proportion of male subjects and NIHSS; (3) the study compared the efficacy and safety of patients with MIS treated with IV-tPA and those not treated with IV-tPA; (4) sufficient data of excellent functional outcome (modified Rankin Scale (mRS) 0-1 or Oxfordshire Handicap Score (OHS) 0-1), sICH and mortality at 3 or 6 months were provided; and (5) onset to needle time was $\leq 4.5$ hours. The following were the exclusion criteria: (1) no comparison group, (2) excellent outcome on hospital discharge, (3) reviews and commentary papers, and (4) studies reporting incomplete data for mentioned subgroups and unavailable data even after exploring the online supplementary materials or contacting the authors.

\section{Data extraction}

Two reviewers (SY and AS) independently extracted the data and compared the results. The characteristics of studies and patients, the diagnostic criteria for MIS, the time from onset to treatment, and the primary outcome (excellent functional outcome defined as mRS $0-1$ or OHS $0-1$ at 3 or 6 months) and secondary outcomes (incidence of sICH and mortality at 3 or 6 months) were extracted. We contacted the authors to obtain further data for one study. ${ }^{11}$

\section{Quality assessment}

The quality of study was assessed using the Newcastle-Ottawa Quality Assessment Scale for cohort studies. ${ }^{23}$ This scale, recommended by the Cochrane Non-Randomized Studies Methods Working Group, is composed of eight items that assess patient selection, study comparability and outcome. Two investigators (SY and WT) performed quality assessment independently, and disagreements were resolved by XW (online supplementary table 1).

\section{Data synthesis and analysis}

We evaluated the heterogeneity among included studies using the $\mathrm{I}^{2}$ test. In the present study, $\mathrm{I}^{2}$ values exceeding $50 \%$ were considered as an indicator of significant heterogeneity. Fixed-effect models were used to estimate the odds ratio(OR) and 95\% confidence interval(CI) if heterogeneity is $<50 \%$; otherwise, random-effect model was used. Begg's funnel plot was used to assess the potential for publication bias. We considered $\mathrm{P}$ values less than 0.05 to be statistically significant. We conducted the meta-analysis using the Cochrane RevMan V.5.3 software and Meta-Analyst (V.3.13beta; Tufts Medical Center). ${ }^{24}$

\section{RESULTS}

\section{Characteristics of included studies}

Among 3818 references obtained by our search strategy, 7 studies (1591 patients) satisfied the eligibility criteria and were included in the final analyses (figure 1). These included five prospective cohort studies and two post-hoc analyses from large clinical trials. The IV-tPA treatment group consisted of 801 patients and the non-treatment group included 790 patients. The baseline characteristics of the included studies are shown in table 1 . The 


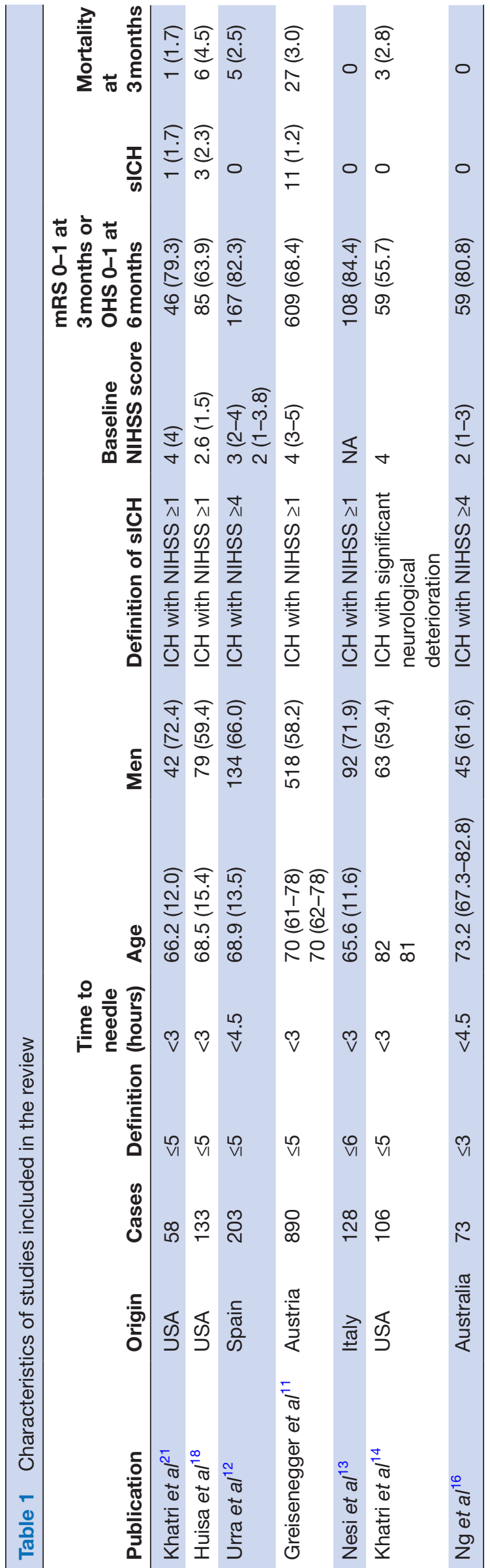

mean age of participants ranged from 66 to 82 years, and the mean baseline NIHSS score ranged from 2 to 4 . The onset to treatment time was recorded in all the studies as less than or equal to 4.5 hours.

ICH, intracranial haemorrhage; mRS, modified Rankin Scale; NA,no data available; NIHSS, National Institutes of Health Stroke Scale; OHS, Oxfordshire Handicap Score; sICH, symptomatic intracranial haemorrhage.

\section{Excellent functional outcome}

All of the included studies report the percentage of mRS $0-1$ or OHS $0-1$ at 3 or 6 months. There are $599(74.8 \%)$ patients with excellent functional outcome among 801 patients treated with IV-tPA and $534(67.6 \%)$ patients in the control group without IV-tPA treatment. The meta-analysis indicated a high odds of excellent outcome $\left(\mathrm{OR}=1.43 ; 95 \%\right.$ CI 1.14 to $\left.1.79 ; \mathrm{P}=0.002, \mathrm{I}^{2}=35 \%\right)$ in patients with MIS treated with IV-tPA compared with those without IV-tPA treatment (figure 2), and no publication bias was observed through the Begg's funnel plot (figure 3).

\section{Safety outcome}

All of the included studies reported on sICH in both comparison groups. In three studies, $15(1.9 \%)$ patients treated with IV-tPA developed sICH, while no sICH was found in the non-treatment group. The meta-analysis demonstrated a significantly higher odds of sICH $\left(\mathrm{OR}=10.13 ; 95 \%\right.$ CI 1.93 to $\left.53.02 ; \mathrm{P}=0.006, \mathrm{I}^{2}=0 \%\right)$ in patients treated with IV-tPA in comparison with those without IV-tPA treatment (figure 4). Only three studies reported sICH and the publication bias was not observed through Begg's funnel plot (online supplementary figure $1)$.

Mortality in patients treated with IV-tPA was 2.4\% ( $\mathrm{n}=19)$, compared with $2.9 \%(n=23)$ in the non-treatment group. The meta-analysis did not identify a significant difference in the odds of mortality $(\mathrm{OR}=0.78$; $95 \%$ CI 0.43 to 1.43 ; $\mathrm{P}=0.43, \mathrm{I}^{2}=0 \%$ ) between the two groups (figure 5). No publication bias was observed through Begg's funnel plot (online supplementary figure 2 ).

\section{DISCUSSION}

The current meta-analysis of the seven included studies found that IV-tPA was associated with excellent functional outcomes at 3 or 6 months following MIS with significantly increased risk of sICH. No significant difference was observed in mortality between patients receiving and not receiving IV-tPA therapy.

MIS is common; about half of ischaemic strokes in the $\mathrm{USA}^{1}$ and approximately $30 \%$ new ischaemic strokes in China are MIS. ${ }^{2}{ }^{3}$ MIS is also associated with significant disability and poor functional outcomes. ${ }^{4-6}$ Previous investigations have found that $28.3 \%$ of patients were not discharged home following MIS, ${ }^{4}$ and $29.0 \%$ of them had significant functional disability at 3 months $(\mathrm{mRS} \geq 2),{ }^{6}$ which may be due to intracranial occlusion, lacunar progression and stroke recurrence. ${ }^{525-27}$ 


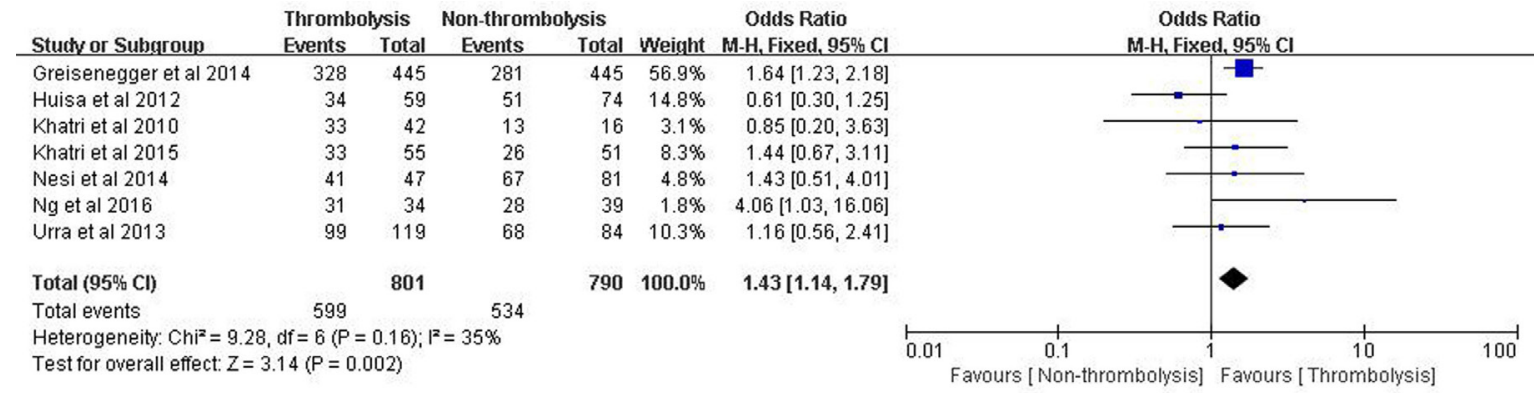

Figure 2 Forest plot for excellent outcome of IV-tPA-treated MIS compared with those without IV-tPA treatment. IVtPA, intravenous recombinant tissue plasminogen activator; $\mathrm{M}-\mathrm{H}$, Mantel-Haenszel; MIS, mild ischaemic stroke.

Currently, approximately $29 \%-32 \%$ of patients with MIS are excluded from IV-tPA therapy due to concerns about sICH. ${ }^{48}$ However, given the considerable longterm morbidity associated with MIS, the role of IV-tPA therapy needed to be closely assessed.

Existing literature regarding the efficacy of IV-tPA therapy in MIS were conflicting. ${ }^{11-22}$ Our meta-analysis suggests that patients treated with IV-tPA were more likely to have excellent functional outcome compared with the non-treatment group, which confirms findings of previous meta-analyses from randomised trials. ${ }^{17}$ In this meta-analysis, $237(67.8 \%)$ patients with MIS (NIHSS $\leq 5)$ in the IV-tPA group and 189 (58.9\%) patients in the control group showed excellent outcome at 3-6 months, and IV-tPA treatment was associated with a 1.48-fold increase in the odds of excellent outcome. ${ }^{17}$ Different baseline characteristics, including baseline NIHSS score and onset to needle time, may have been the underlying reasons for the no benefit found in patients with MIS treated with IV-tPA in some studies. The Huisa et $a l^{18}$ study indicated that patients with MIS treated with IV-tPA had similar outcomes as patients not receiving IV-tPA treatment. In this study, the mean baseline NIHSS score was 3.4 in patients treated with IV-tPA and significantly higher than untreated patients (the mean baseline NIHSS score is 1.9) ${ }^{18}$ Data from the main paper of

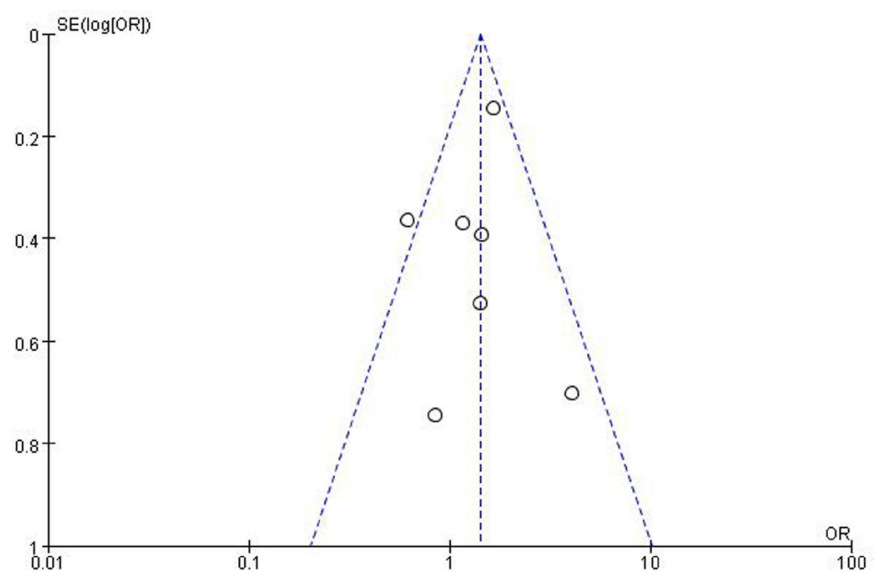

Figure 3 Funnel plot for publication bias for excellent outcome of intravenous recombinant tissue plasminogen activator-treated mild ischaemic stroke. the Third International Stroke Trial-3, in which the onset to needle time in some patients is more than 4.5 hours, suggested IV-tPA treatment had no benefits for patients with MIS. ${ }^{29}$ However, if restricted patients met the standard treatment criteria, including randomisation within 3 hours, IV-tPA treatment has a significant benefit for patients with MIS. ${ }^{14}$

The risk of haemorrhage is one of the major reasons that patients with MIS are excluded from IV-tPA treatment. In our meta-analysis, 15 (1.9\%) patients developed sICH, and all of these patients received IV-tPA therapy. Our results were in line with previous studies ${ }^{5} 3031$ showing that the risk of sICH was low for patients with MIS treated with IV-tPA, although higher in comparison with the non-treatment group. A meta-analysis of 12 trials reported that the risk of sICH in all patients with stroke was $7.7 \% .^{30}$ The incidence of sICH based on stroke severity was compared in a cohort study that found the risk of haemorrhage was about $2 \%$ in patients with MIS and $8.1 \%$ in patients with a baseline NIHSS score of more than $6 .{ }^{31}$ Moreover, we found that in all 15 patients with sICH reported in three studies, defined as any ICH with NIHSS score increase of $\geq 1,{ }^{11} 1821$ haemorrhage was not serious and may not be associated with disability and mortality. The mortality rate of IV-tPA-treated patients $(2.4 \%)$ and non-tPA-treated patients $(2.9 \%)$ was similar, which suggested IV-tPA treatment did not affect mortality. Our meta-analysis, along with previous studies, suggests the overall risk of sICH is low and does not contribute to disability or mortality in patients with MIS treated with IV-tPA. The results of our meta-analysis support the need for ongoing randomised trials to further investigate the role of IV-tPA therapy in MIS. The TNK-Tissue-Type Plasminogen Activator Evaluation for Minor Ischemic Stroke With Proven Occlusion-2 (TEMPO-2) and A Study of the Efficacy and Safety of Activase (Alteplase) in Patients With Mild Stroke (PRISMS) are two ongoing randomised, placebo-controlled trials evaluating the efficacy, and particularly the safety, of IV-tPA or Tenecteplase -tissuetype plasminogen activator in patients with MIS (http:// clinicaltrials.gov/).

There were some limitations to our meta-analysis. First, most of the studies included fairly small number of subjects, together with potential bias arising from the 


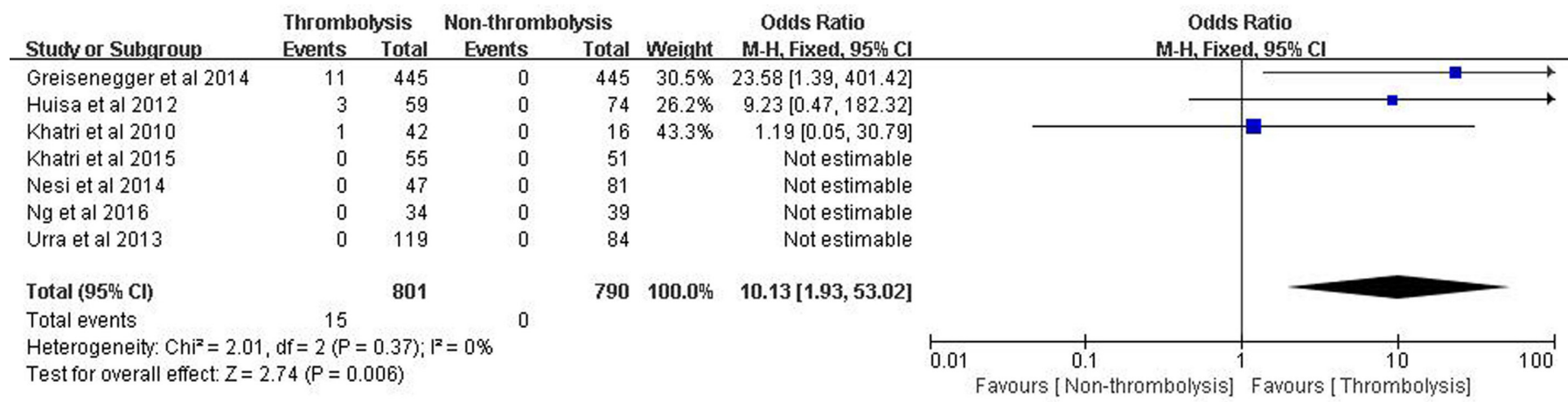

Figure 4 Forest plot for symptomatic intracranial haemorrhage of IV-tPA-treated mild ischaemic stroke compared with those without IV-tPA treatment. IV-tPA, intravenous recombinant tissue plasminogen activator; M-H, Mantel-Haenszel.

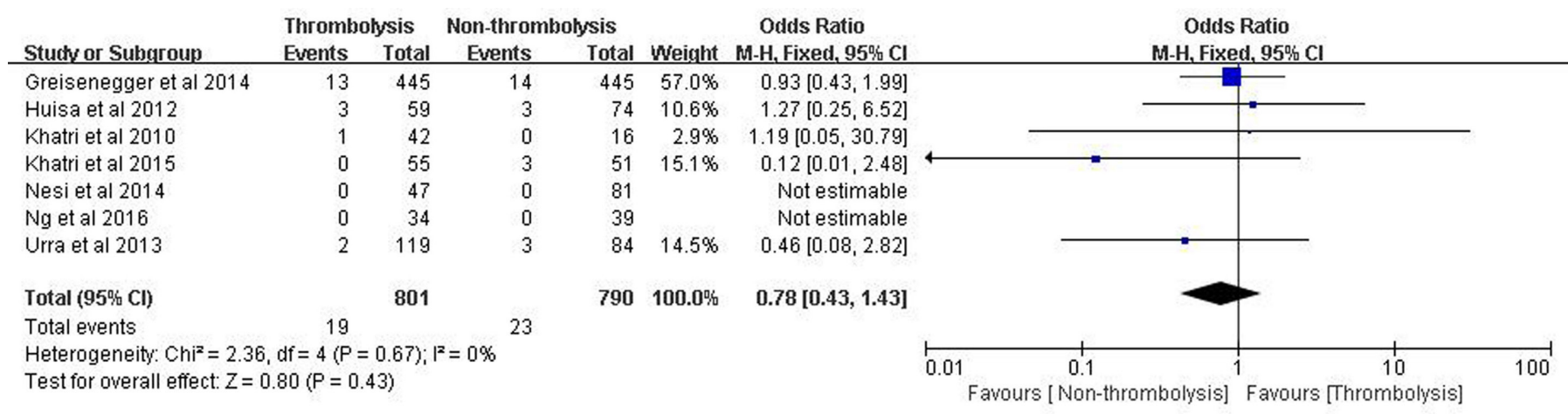

Figure 5 Forest plot for mortality of IV-tPA-treated mild ischaemic stroke compared with those without IV-tPA treatment. IVtPA, intravenous recombinant tissue plasminogen activator; $\mathrm{M}-\mathrm{H}$, Mantel-Haenszel .

non-randomised nature of observational studies. This bias could not be compensated for satisfactorily, and the unadjusted results from observational studies remain less conclusive even when the data from post-hoc analyses of large randomised controlled trial are combined. Second, we could not report the results based on adjusted OR as four of the included studies did not report these data. Third, the definition of sICH was different among included studies.

\section{CONCLUSION}

In conclusion, our meta-analysis found that patients with MIS treated with IV-tPA had improved functional outcome compared with patients without IV-tPA treatment. Although IV-tPA treatment increased the risk of sICH, this risk is low and did not affect mortality. Our results suggest IV-tPA treatment is beneficial and relatively safe in patients with MIS, and there is a need for further randomised clinical trials to confirm these findings.

\section{Author affiliations}

${ }^{1}$ Department of Neurology and Suzhou Clinical Research Center of Neurological

Disease, The Second Affiliated Hospital of Soochow University, Suzhou, China

${ }^{2}$ The George Institute for Global Health, University of New South Wales, Sydney, New South Wales, Australia

${ }^{3}$ Department of Neurology, Hospital Kuala Lumpur, Kuala Lumpur, Malaysia

${ }^{4}$ Department of Neurology, Suzhou Hospital of Traditional Chinese Medicine, Suzhou, China

${ }^{5}$ Institutes of Neuroscience, Soochow University, Suzhou, China
Contributors $\mathrm{YC}, \mathrm{XW}$ and $\mathrm{C}-\mathrm{FL}$ conceived this meta-analysis and revised the manuscript. XW searched the database. SY and AS collected and extracted the data. SY and WT conducted statistical analysis. SY and QH drafted this manuscript.

Funding This work was supported in part by grants from the National Natural Science Foundation of China (81471195), Suzhou Clinical Research Center of Neurological Disease (Szzx201503) and Jiangsu Provincial Medical Key Discipline Project, The Second Affiliated Hospital of Soochow University Preponderant Clinic Discipline Group Project Funding (XKQ2015002). This was also partly supported by the Priority Academic Program Development of Jiangsu Higher Education Institutions (PAPD).

Competing interests None declared.

Provenance and peer review Not commissioned; externally peer reviewed. Data sharing statement No additional data are available.

Open Access This is an Open Access article distributed in accordance with the Creative Commons Attribution Non Commercial (CC BY-NC 4.0) license, which permits others to distribute, remix, adapt, build upon this work non-commercially, and license their derivative works on different terms, provided the original work is properly cited and the use is non-commercial. See: http://creativecommons.org/ licenses/by-nc/4.0/

(C) Article author(s) (or their employer(s) unless otherwise stated in the text of the article) 2018. All rights reserved. No commercial use is permitted unless otherwise expressly granted.

\section{REFERENCES}

1. Reeves M, Khoury J, Alwell K, et al. Distribution of national institutes of health stroke scale in the cincinnati/Northern Kentucky stroke study. Stroke 2013;44:3211-3.

2. Wang YL, Wu D, Liao X, et al. Burden of stroke in China. Int J Stroke 2007;2:211-3. 
3. Zhao D, Liu J, Wang W, et al. Epidemiological transition of stroke in China: twenty-one-year observational study from the Sino-MONICABeijing Project. Stroke 2008;39:1668-74.

4. Smith EE, Fonarow GC, Reeves MJ, et al. Outcomes in mild or rapidly improving stroke not treated with intravenous recombinant tissue-type plasminogen activator: findings from get with the guidelines-stroke. Stroke 2011;42:3110-5.

5. Coutts SB, Modi J, Patel SK, et al. What causes disability after transient ischemic attack and minor stroke?: Results from the CT and MRI in the triage of TIA and minor cerebrovascular events to identify high risk patients (CATCH) study. Stroke 2012;43:3018-22.

6. Khatri P, Conaway MR, Johnston KC. Ninety-day outcome rates of a prospective cohort of consecutive patients with mild ischemic stroke. Stroke 2012;43:560-2.

7. Jauch EC, Saver JL, Adams HP, et al. Guidelines for the early management of patients with acute ischemic stroke: a guideline for healthcare professionals from the American heart association/ American stroke association. Stroke 2013;44:870-947.

8. European Stroke Organisation (ESO) Executive CommitteeESO Writing Committee. Guidelines for management of ischaemic stroke and transient ischaemic attack 2008. Cerebrovasc Dis 2008;25:457-507.

9. Minematsu K, Toyoda K, Hirano T, et al. Guidelines for the intravenous application of recombinant tissue-type plasminogen activator (alteplase), the second edition, October 2012: a guideline from the Japan stroke society. J Stroke Cerebrovasc Dis 2013;22:571-600.

10. Casaubon LK, Boulanger JM, Blacquiere D, et al. Canadian stroke best practice recommendations: hyperacute stroke care guidelines, update 2015. Int J Stroke 2015;10:924-40.

11. Greisenegger S, Seyfang L, Kiechl S, et al. Thrombolysis in patients with mild stroke: results from the Austrian stroke unit registry. Stroke 2014:45:765-9.

12. Urra X, Ariño $\mathrm{H}$, Llull $\mathrm{L}$, et al. The outcome of patients with mild stroke improves after treatment with systemic thrombolysis. PLOS One 2013;8:e59420.

13. Nesi M, Lucente G, Nencini P, et al. Aphasia predicts unfavorable outcome in mild ischemic stroke patients and prompts thrombolytic treatment. J Stroke Cerebrovasc Dis 2014;23:204-8.

14. Khatri P, Tayama D, Cohen G, et al. Effect of intravenous recombinant tissue-type plasminogen activator in patients with mild stroke in the third international stroke trial-3: post hoc analysis. Stroke 2015;46:2325-7.

15. Yeo LLL, Ho R, Paliwal $P$, et al. Intravenously administered tissue plasminogen activator useful in milder strokes? A meta-analysis. $J$ Stroke Cerebrovasc Dis 2014;23:2156-62.

16. Ng FC, Coote S, Frost T, et al. Utility of computed tomographic perfusion in thrombolysis for minor stroke. Stroke 2016;47:1914-6.

17. Emberson J, Lees KR, Lyden P, et al. Effect of treatment delay, age, and stroke severity on the effects of intravenous thrombolysis with alteplase for acute ischaemic stroke: a metaanalysis of individual patient data from randomised trials. Lancet 2014;384:1929-35.

18. Huisa BN, Raman R, Neil W, et al. Intravenous tissue plasminogen activator for patients with minor ischemic stroke. J Stroke Cerebrovasc Dis 2012;21:732-6.

19. Spokoyny I, Raman R, Ernstrom K, et al. Defining mild stroke: outcomes analysis of treated and untreated mild stroke patients. J Stroke Cerebrovasc Dis 2015;24:1276-81.

20. National Institute of Neurological Disorders Stroke rt-PA Stroke Study Group. Recombinant tissue plasminogen activator for minor strokes: the national institute of neurological disorders and stroke rt-PA stroke study experience. Ann Emerg Med 2005;46:243-52.

21. Khatri $\mathrm{P}, \mathrm{Kleindorfer} \mathrm{DO}$, Yeatts $\mathrm{SD}$, et al. Strokes with minor symptoms: an exploratory analysis of the national institute of neurological disorders and stroke recombinant tissue plasminogen activator trials. Stroke 2010;41:2581-6.

22. Shi L, Zhang M, Liu H, et al. Safety and outcome of thrombolysis in mild stroke: a meta-analysis. Med Sci Monit 2014;20:2117-24.

23. Wells gA, Shea B, O'Connell D, et al. The newcastle-ottawa scale (NOS) for assessing the quailty of nonrandomised studies in metaanalyses. $2009 \mathrm{Http}: / / w w w$ ohri ca/programs/clinical_epidemiology/ oxford htm

24. Wallace BC, Schmid CH, Lau J, et al. Meta-analyst: software for meta-analysis of binary, continuous and diagnostic data. BMC Med Res Methodol 2009;9:80.

25. Nedeltchev K, Schwegler B, Haefeli T, et al. Outcome of stroke with mild or rapidly improving symptoms. Stroke 2007;38:2531-5.

26. Horton M, Modi J, Patel SK, et al. Refinement of imaging predictors of recurrent events following transient ischemic attack and minor stroke. PLoS One 2013;8:e65752.

27. Wang $\mathrm{Y}$, Wang $\mathrm{Y}$, Zhao $\mathrm{X}$, et al. Clopidogrel with aspirin in acute minor stroke or transient ischemic attack. N Engl J Med 2013;369:11-19.

28. Adeoye O, Hornung R, Khatri P, et al. Recombinant tissue-type plasminogen activator use for ischemic stroke in the United States: a doubling of treatment rates over the course of 5 years. Stroke 2011;42:1952-5.

29. Sandercock P, Wardlaw JM, Lindley RI, et al. The benefits and harms of intravenous thrombolysis with recombinant tissue plasminogen activator within $6 \mathrm{~h}$ of acute ischaemic stroke (the third international stroke trial [IST-3]): a randomised controlled trial. Lancet 2012;379:2352-63.

30. Wardlaw JM, Murray V, Berge E, et al. Recombinant tissue plasminogen activator for acute ischaemic stroke: an updated systematic review and meta-analysis. Lancet 2012;379:2364-72.

31. Strbian D, Piironen K, Meretoja A, et al. Intravenous thrombolysis for acute ischemic stroke patients presenting with mild symptoms. Int $J$ Stroke 2013;8:293-9. 